J. Clin. Chem. Clin. Biochem.

Vol. 18, 1980, pp. 175-181

\title{
Die prognostische Bedeutung von zirkulierenden Immunkomplexen des Carcinoembryonalen Antigens (CEA) bei Patienten mit Adenokarzinomen des Gastrointestinaltraktes
}

\author{
Von H. J. Staab, F. A. Anderer \\ Friedrich-Miescher-Laboratorium der Max-Planck-Gesellschaft, Tübingen,
}

\author{
E. Stumpf und R. Fischer \\ Chirurgische Klinik, Stuttgart - Bad Cannstatt
}

(Eingegangen am 27. Juli/10. Oktober 1979)

Zusammenfassung: Bei 350 Patienten mit histologisch gesicherten Adenokarzinomen des Gastrointestinaltraktes wurde präoperativ die Konzentration der CEA-Immunkomplexe neben freiem CEA bestimmt. Bei 86 Patienten (25\%) ließen sich zirkulierende CEA-Immunkomplexe nachweisen, wobei mit zunehmender Tumorausdehnung nach der TNMKlassifikation auch der Anteil der Patienten mit CEA-Immunkomplexen zunahm. 74/86 Patienten hatten gleichzeitig pathologisch erhöhte Konzentrationen an freiem CEA. In der postoperativen Überwachung konnte die Bestimmung von zirkulierenden CEA-Immunkomplexen für prognostische Aussagen verwertet werden. Bei 30/50 Rezidivpatienten wurden spätestens zum Zeitpunkt der klinischen Diagnose CEA-Immunkomplexe festgestellt. Das Auftreten der CEAImmunkomplexe kann zur Charakterisierung des Immunstatus herangezogen werden. Bei einem Teil der Patienten mit weit fortgeschrittenen Stadien des Tumorwachstums war einige Monate vor dem Exitus ein starker Anstieg der CEA-Immunkomplexe beobachtet worden (44\% der Patienten). In der Präfinalphase ist bei $13 \%$ der Patienten die Konzentration der CEA-Immunkomplexe wieder weitgehend abgefallen.

\section{Prognostic value of circulating immune complexes of carcinoembryonic antigen (CEA) in patients with adenocar- cinoma of the gastrointestinal tract}

Summary: CEA immune complexes and unbound CEA were preoperatively determined in 350 patients with histologically confirmed adenocarcinoma of the gastrointestinal tract. Circulating CEA immune complexes could be detected in 86 patients (25\%) where an increase of tumor extension according to TNM classification was concomitant with an increasing percentage of patients with CEA immune complexes. 74/86 patients showed simultaneously pathological concentrations of unbound CEA. During postoperative surveillance the determinations of circulating CEA immune complexes could be used as prognostic criteria. In 30/50 patients with recurrent cancer CEA immune complexes were detected latest at the time of clinical diagnosis. Appearance of CEA immune complexes might contribute to characterization of the immune status of the patients. Some of the patients with widespread tumors exhibited a rapid increase of CEA immune complexes a few months before exitus (44\% of the patients). Before exitus $13 \%$ of the patients again showed a greatly decreased concentration of CEA-immune complexes.

\section{Einführung}

Die postoperative Úberwachung von Patienten mit Adenokarzinomen des Gastrointestinal träktes durch Routinekontrollen der Konzentration des carcinoembryonalen Antigens (CEA) im Serum (1) hat in den letzten Jahren seinen Wert als Diagnosehilfe bei Rezidiven bestätigt $(2-12)$. In den meisten Fällen konnte ein weiteres Tumorwachstum auf der Basis des CEA-Anstiegs nicht nur bis zu 12 Monaten früher erkannt werden, sondern es wurden auch die methodischen Grundlagen ausgearbeitet, die eine Differenzierung von lokal begrenzten Rezidiven und metastasierendem Tumorwachstum durch Verlaufsbeurteilung des CEA-Anstiegs möglich machte (13-16).

Uber den Immunstatus von Patienten mit pathologisch erhöhter CEA-Konzentration ist wenig bekannt. Nach den Ergebnissen von Hellström et al. (17) kann auch bei einer 
starken zellvermittelten Anti-Tumor-Immunität progressives Tumorwachstum stattfinden, wenn die Zerstörung der Tumorzellen durch Immunlymphocyten von "Rezeptorblockierenden Antikörpern" verhindert wird. Verschiedene andere Untersuchungen (18-21) geben Anlaß zur Annahme, daß diese "blockierenden Antikörper" ImmunKomplexe sein können. Unter diesem Aspekt ist es von großer Bedeutung, ob CEA, insbesondere in pathologischen Konzentrationen, die Bildung von spezifischen Antikörpern induzieren kann. Bisher konnten in einigen ausgewählten Seren von Patienten mit Adenokarzinomen des Gastroin testinaltraktes CEA-bindende Immunglobuline $(22,23)$ und schließlich auch CEA-Immunkomplexe $(24,25)$ nachgewiesen werden. Aufgrund der geringen Patientenzahlen und fehlender klinischer Daten ist jedoch eine eindeutige Korrelierung von progressivem Tumorwachstum mit der Anwesenheit von zirkulierenden CEA-Immunkomplexen nicht möglich gewesen.

In der hier vorgelegten Studie wurde im Rahmen der postoperativen Uberwachung von Patienten mit histologisch gesicherten Adenokarzinomen des Gastrointestinal traktes (11) untersucht, inwieweit das präoperative Auftreten zirkulierender CEA-Immunkomplexe an bestimmte klinische Stadien der Tumorentwicklung zum Zeitpunkt der Operation gebunden ist. Darüber hinaus wurden während der postoperativen Überwachung die CEA-Immunkomplexe neben freiem CEA in zeitlichen Abständen von 2-3 Monaten in den Patientenseren bestimmt, um insbesondere in den Fällen einer Rezidivierung oder sekundären Metastasierung mögliche $\mathrm{Zu}$ sammenhänge charakterisieren zu können.

\section{Methoden und Patienten}

Der Gehalt der Patientenseren an freiem CEA wurde nach Perchlorsäureextraktion radioimmunologisch mit dem CEARoche-RIA-Test nach der Hansen'schen Z-Gelmethode (26) analysiert. Neuere Untersuchungen (27) haben gezeigt, dals das in diesem RIA-test benützte Testserum nur unwesentlich mit der CEA-verwandten Komponente CCA-III (colon carcinoma antigen III) - einem mit NCA (nonspecific cross-reacting antigen) identischen Glykoprotein (28) - kreuzreagiert. Die CEAImmunkomplexe finden sich ausschließlich in den Sedimenten der Perchlorsäureextraktion, vorausgesetzt, daß die Verdünnung

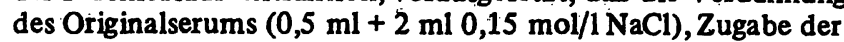
Perchlorsäure und Sedimentation des Präzipitats $(2500 \mathrm{~g}$; $20 \mathrm{~min}$ ) ohne Verzögerung durchgeführt werden, und somit eine partielle Dissoziation der CEA-Immunkomplexe verhindert wird. Der CEA-Gehalt der Sedimente kanin näch einer bereits beschriebenen Methode (25) ebenfalls radioimmunologisch bestimmt werden, indem die Sedimente in $2 \mathrm{ml} 2 \mathrm{~mol} / \mathrm{l}$ Tris gelöst (pH 9,0-9,5), davon $0,5 \mathrm{ml}$ mit physiol. $\mathrm{NaCl}$ auf $5 \mathrm{ml}$ verdünnt und ohne weitere Perchlorsäureextraktion für den Radioimmuntest dialysiert werden. Die Signifikanzgrenze liegt bei der Bestimmung des freien CEA bei $2 \mu \mathrm{g} / 1$ Serum (11) und bei der Bestimmung des in Immunkomplexen gebundenen CEA, bedingt durch die Verdünnungsstufen, bei 3,5 $\mu \mathrm{g} / \mathrm{l}$ Serum.

Die optimale Trennung des freien CEA von den CEA-Immunkomplexen war von der Gesamtkonzentration des Serums abhängig (25). Vorverdünnungen der Serèn müssen daher mit einem entsprechenden Kontrollserum gemacht werden.

Eine unspezifische Kopräzipitation und Einschlüsse von freiem CEA im Perchlorsäure-Sediment wurden durch Zumischen bestimmter Mengen von exogenem, radioaktiv mạkiertem CEA zu einem Kontrollserum untersucht. Bei CEA-Konzentrationen .zwischen 30 und $1000 \mu \mathrm{g} / 1$ Serum wurden zwischen $3-10 \%$ des freien CEA im Perchlorsäurepräzipitat gefunden. Der relative Signifikanzbereich für CEA-Immunkomplexe muß also über $10 \%$ der Menge an freiem CEA liegen.

Die bisherige Studie erfaßte 350 Patienten, bei denen präoperativ das freie CEA neben CEA-Immunkomplexen bestimmt wurde. Davon sind 139 weiblich und 211 männlich mit folgender Altersverteilung: bis 50 Jahre $n=46,50-60$ Jahre $n=63,60-70$ Jahre $\mathrm{n}=116$ und über 70 Jahre $\mathrm{n}=125$.

Zur Bestimmung der präoperativen CEA-Werte wurden Blutproben wenige Tage vor der Operation entnommen. Die weitere Uberwachung der Patienten unterlag den gleichen Einbestellungskriterien, die für unsere Langzeitstudie zur postoperativen Überwachung von Patienten mit Gastrointestinalkarzinomen spezifiziert wurde (11).

\section{Ergebnisse}

\section{Patienten mit CEA-İmmunkomplexen}

Durch die präoperative Bestimmung der CEA-Immunkomplexe im Serum der Patienten mit Adenokarzinomen des Gastrointestinaltraktes sollte zunächst geklärt werden, ob sich die Erfassungsquoten zugunsten CEA-positiver Fälle erhöhen. Tabelle 1, Spalte 5, zeigt für die gegenwärtige Patientengruppe die Erfassungsquoten auf der Basis der präoperativen Serumkonzentration an freiem CEA, die nur unwesentlich von unseren früheren Werten

Tab. 1. Übersicht über die bisher erfaßten Patienten mit Adenokarzinomen des Gasstrointestinaltraktes, aufgeschlüsselt nach Karzinomspezifikation ( $\mathrm{n}=$ Patientenzahl) und nach \%-Anteilen der Patienten, bei denen präoperativ

a) CEA-Immunkomplexe festgestellt wurden,

b) Patienten mit CEA-Immunkomplexen, jedoch mit einer Konzentration an freiem CEA im Normalbereich, und

c) Patienten, deren Konzentration an freiem CEA über dem Normalbereich lag. Konzentrationsangaben beziehen sich auf CEA in $\mu \mathrm{g} / \mathrm{l}$ Serum.

\begin{tabular}{|c|c|c|c|c|}
\hline \multirow{3}{*}{ Karzinomspezifikation } & \multirow[b]{3}{*}{$\mathrm{n}$} & \multirow{2}{*}{$\begin{array}{l}\text { a } \\
\text { CEA-Immun- } \\
\text { komplexe } \\
>3,5 \mu \mathrm{g} / \mathrm{l} \mathrm{CEA}\end{array}$} & \multirow{2}{*}{$\begin{array}{l}\mathrm{b} \\
\text { CEA-Immunkomplexe } \\
>3,5 \mu \mathrm{g} / 1 \mathrm{CEA} \\
\text { freies CEA }<2 \mu \mathrm{g} / 1\end{array}$} & \multirow{2}{*}{$\begin{array}{l}\text { c } \\
\text { freies CEA } \\
>2 \mu \mathrm{g} / 1\end{array}$} \\
\hline & & & & \\
\hline & & $\%$ & $\%$ & $\%$ \\
\hline $\begin{array}{l}\text { Magen } \\
\text { Pankreas } \\
\text { Colon (asc., transv., desc.) } \\
\text { Colon sigmoideum } \\
\text { Rektum }\end{array}$ & $\begin{array}{r}139 \\
18 \\
43 \\
73 \\
77\end{array}$ & $\begin{array}{l}20,1 \\
44,4 \\
23,2 \\
36,9 \\
22,0\end{array}$ & $\begin{array}{l}4,3 \\
0 \\
9,3 \\
1,4 \\
2,6\end{array}$ & $\begin{array}{l}54,6 \\
77,7 \\
62,7 \\
67,1 \\
71,4 \quad \ldots\end{array}$ \\
\hline
\end{tabular}


(11) abweichen. Es konnte angenommen werden, daß bei manchen Patienten die Konzentration an freiem CEA nur deshalb im Normalbereich lag, weil das CEA durch Antikörper gebunden war. Die Spalte 4 gibt den Anteil der Patienten wieder, der präoperativ nur in der Fraktion der Immunkomplexe pathologische CEA-Konzentrationen aufwies. Eine nennenswerte Erhöhung der Erfassungsquoten wird nur bei Adenokarzinomen des Magens $(+4.3 \%)$ und des Colon ascendens, transversum und descendens $(+9,3 \%)$ erreicht. Der Gesamtanteil der Patienten mit präoperativen CEA-Immunkomplexen (Tab. 1, Spalte 3) ist bei den verschiedenen Karzinomspezifikationen etwa gleich mit der Ausnahme von Adenokarzinomen des Colon sigmoideum und des Pankreas, die einen nahezu doppelt so hohen Anteil aufweisen.

\section{Korrelierung der TNMStadien mit dem präoperativen Auftreten von CEA-Immunkomplexen}

Die Vermutung, daß bestimmte klinische Stadien der Karzinome direkt oder indirekt mit dem präoperativen Auftreten von CEA-Immunkomplexen bzw. CEA-bindender Immunglobuline verknüpft sind, läßt sich am einfachsten durch Korrelierung mit der Klassifizierung nach dem TNM-System $(29,30)$, d.h. mit den allgemein üblichen Definitionen der lokalen Ausdehnung maligner Tumoren, prüfen. In Tabelle 2 sind die Patienten nach ihrer TNM-Klassifikation aufgegliedert und fün verschiedenen CEA-Kriterien zugeordnet worden. Die Patienten in Zeile 1 hatten keine CEA-Immunkomplexe und kein freies CEA bzw. eine CEA-Konzentration im Serum, die im Normalbereich lag. Diese Gruppe umfaßt 125 Patienten, d.h. 35,7\%, was mit dem früher berichteten Anteil der CEA-freien Patienten (11) gut übereinstimmt. Die Verteilung dieser Patienten auf die drei verschiedenen TNM-Klassen dient für die Beurteilung der weiteren Patientengruppen als Bezugssystem. Als Vergleichsgröße kann das Verhältnis $P$ der Patienten ohne Metastasen $(\mathrm{N} \equiv \mathrm{O} ; \mathrm{M}=0)$ und der mit Metastasen ( $\mathrm{N}$ und/oder $\mathrm{M} \neq 0$ ) herangezogen werden, also in dieser Gruppe $P=1: 1$. Bei den Patienten in Tabelle 2, Zeile 3, konnten ebenfalls keine CEA-Immunkomplexe nachgewiesen werden, jedoch hatten sie alle pathologisch erhöhte Konzentrationen an freiem CEA. In dieser Gruppe $(n=139)$ ist der Anteil der Patienten mit regionalen Lymphknoten $(N \neq 0)$ und Fernmetastasen $(M \neq 0)$ wesentlich größer und es errechnet sich ein Patientenverhältnis $P=1: 1,8$. In den Patientengruppen mit CEA-Immunkomplexen verschiebt sich dieses Verhältnis noch weiter zu ungunsten der metastasenfreien Patienten (Tab. 2, Zeile 2, 4, 5; $n=86$ ). Bei

Patienten mit CEA-Immunkomplexen und pathologischen Konzentrationen an freiem CEA wird sogar ein Verhältnis $P=1: 6$ erreicht. Diese weitgehende Korrelierbarkeit der klinischen Stadien der Karzinome mit dem präoperativen Auftreten von CEA-Immunkomplexen kann daher für eine prognostische Aussage herangezogen werden.

Es stellt sich nun die Frage, ob der prognostische Wert präoperativ festgestellter CEA-Immunkomplexe für alle Karzinomspezifikationen gleich ist. In Tabelle 3 ist eine entsprechende Auswertung vorgenommen worden, für die nur die Patientengruppen erfaßt wurden, die pathologische Konzentrationen an freiem CEA oder/und CEA-Immunkomplexen aufwiesen $(n=225)$. Für jede Karzinomspezifikation wurde der prozentuale Anteil der Patienten mit CEA-Immunkomplexen innerhalb der drei TNM-Klassen ermittelt. Dabei zeigte sich, daß bei Adenokarzinomen des Colon ascendens, transversum und descendens, des Magens und des Rektum das präoperative Auftreten von CEA-Immunkomplexen eine ungünstige Prognose anzeigt. Im klinischen Stadium, in dem noch keine Metastasierung festgestellt werden konnte, hatten maximal 15\% der Patienten CEAImmunkomplexe, bei Fernmetastasen 52 bis $60 \%$. Anders verhält es sich bei Karzinomen des Colon sigmoideum und des Pankreas. Hier hatte nahezu die Hälfte der Patienten CEA-Immunkomplexe ohne zunächst feststellbare Metastasierung. Man müßte jedoch annehmen, daß Lymphknotenmetastasen vorhanden waren, die intraoperativ nicht erkannt werden konnten, da z.B. bei typischer 3-zeitiger Sigmaresektion das Retroperitoneum nicht eröffnet wird.

Tab. 2. Aufgliederung der Patienten entsprechend der klinischen Stadien der Tumorausdehnung (TNM-Klassifikation) und Zuordnung verschiedener Konzentrationsbereiche des freien CEA und der CEA-Immunkomplexe (präoperative Werte).

\begin{tabular}{|c|c|c|c|c|c|}
\hline \multicolumn{2}{|c|}{ 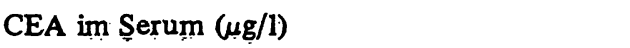 } & \multicolumn{4}{|c|}{ Patienten klassifiziert nach } \\
\hline freies CEA & CEA-Immunkomplexe & $\begin{array}{l}T \neq 0 \\
N=0 \\
M=0\end{array}$ & $\begin{array}{l}T \neq 0 \\
N \neq 0 \\
M=0\end{array}$ & $\begin{array}{l}T \neq 0 \\
N \neq 0 \\
M \neq 0\end{array}$ & gesamt \\
\hline $\begin{array}{l}0-2 \\
0-2 \\
>2 \\
>2 \\
>2\end{array}$ & $\begin{array}{c}0-3,5 \\
>3,5 \\
0-3,5 \\
>3,5 a \\
>3,5\end{array}$ & $\begin{array}{r}63 \\
3 \\
50 \\
8 \\
3\end{array}$ & $\begin{array}{r}48 \\
4 \\
59 \\
23 \\
7\end{array}$ & $\begin{array}{r}14 \\
5 \\
30 \\
27 \\
6\end{array}$ & $\begin{array}{r}125 \\
12 \\
139 \\
58 \\
16\end{array}$ \\
\hline Patienten & & 127 & 141 & 82 & 350 \\
\hline
\end{tabular}

afreies.CEA höher als.CEA-Immunkomplexe

bCEA-Immunkomplexe höher als freies CEA 
Tab. 3. Prozentanteile der Patienten mit präoperativen CEAImmunkomplexen $(n=86)$ innerhalb der Patientengruppe mit präoperativ pathologischen CEA-Konzentrationen $(n=225)$ aufgegliedert nach Karzinomspezifikation und TNM-Klassifikation.

\begin{tabular}{|c|c|c|c|c|c|c|}
\hline \multirow[t]{2}{*}{ Karzinomspezifikation } & \multirow{2}{*}{\multicolumn{2}{|c|}{$\begin{array}{c}T \neq 0 \\
N=0 \\
M=0\end{array}$}} & \multirow{2}{*}{\multicolumn{2}{|c|}{$\begin{array}{r}\mathrm{T} \neq 0 \\
\mathrm{~N} \neq 0 \\
\mathrm{M}=0\end{array}$}} & \multicolumn{2}{|c|}{$\begin{array}{l}T \neq 0 \\
N \neq 0 \\
M \neq 0\end{array}$} \\
\hline & & & & & $\mathbf{n}$ & $\%$ \\
\hline Magen & $2 / 15$ & 13 & $13 / 4$ & 4132 & $12 / 23$ & 52 \\
\hline Pankreas & $4 / 9$ & 44 & - & - & $4 / 4$ & 100 \\
\hline $\begin{array}{l}\text { Colon (asc., transv., } \\
\text { desc.) }\end{array}$ & $0 / 9$ & 0 & $6 / 1$ & 1638 & $4 / 8$ & 50 \\
\hline Colon sigmoideum & $5 / 12$ & 42 & . $8 / 1$ & 1554 & $12 / 20$ & 59 \\
\hline Rektum & $3 / 20$ & 15 & $7 / 2$ & 2331 & $6 / 10$ & 60 \\
\hline
\end{tabular}

\section{CEA-Verlaufskontrollen bei Patienten mit CEA-Immun- komplexen}

Die Patientenüberwachung durch Routinebestimmungen des freien CEA im Serum ermöglichte bisher frühzeitige Aussagen über Rezidivierung und Metastasierung $(11,13$, 16). Es war zu erwarten, daß durch die zusätzliche Bestimmung der CEA-Immunkomplexe insbesondere Informationen über den Immunstatus der Patienten zugänglich werden. Die Verlaufskurven, die für die CEAImmunkomplexe erhalten wurden, verliefen nur bei einem Teil der Fälle analog zu den Kurven des freien CEA. Als Beispiel sind in Abbildung 1 a die CEA-Kurven= verläufe für einen Patienten wiedergegeben, der präoperativ erhöhte Werte an freiem CEA und CEA-Immunkomplexen hatte, aber nach der Operation nur noch Werte im Normalbereich aufwies. Während der postoperativen Überwachung stiegen die Werte für freies CEA und für CEAImmunkomplexe wieder an und nach 15 Monaten wurden klinisch Lebermetastasen festgestellt. Abweichend von diesem Analogverhalten der CEA-Kurven sind zeitlich befristete Erhöhungen der CEA-Immunkomplexe, die jedoch nicht immer von einem gleichzeitigen Ansteigen des freien CEA begleitet sein müssen. In Abbildung $1 \mathrm{~b}$ ist ein Beispiel für einen operationsinduzierten Anstieg der CEA-Immunkomplexe und des freien CEA abgebil$\operatorname{det}(13 / 86$ CEA-Immunkomplex-positive Fälle). Die Patientengruppe mit diesem Verlaufskriterium setzt sich zu 70\% aus Patienten mit Lymphknotenmetastasen und $\mathrm{zu} 30 \%$ aus Patienten ohne erkennbare Metastasierung zum Zeitpunkt der Operation zusammen. Ein befristeter Anstieg der CEA-Immunkomplexe ist auch bei manchen Patienten (6/86) zu einem späterèn Zeitpunkt während der postoperativen Ubberwachung beobachtet worden (Abb. 1c). Die Urșache für diese Zunahme der CEA-Immunkomplexe konnte bis jetzt nicht eindeutig geklärt werden. Es ist jedoch denkbar, daß bestimmte entzündliche Prozesse unabhängig vom Stadium des Karzinoms zu diesem Phänomen führen können. Bei zwei dieser Patienten wurde zum fraglichen Zeitpunkt eine Blasenentzündung festgestellt.

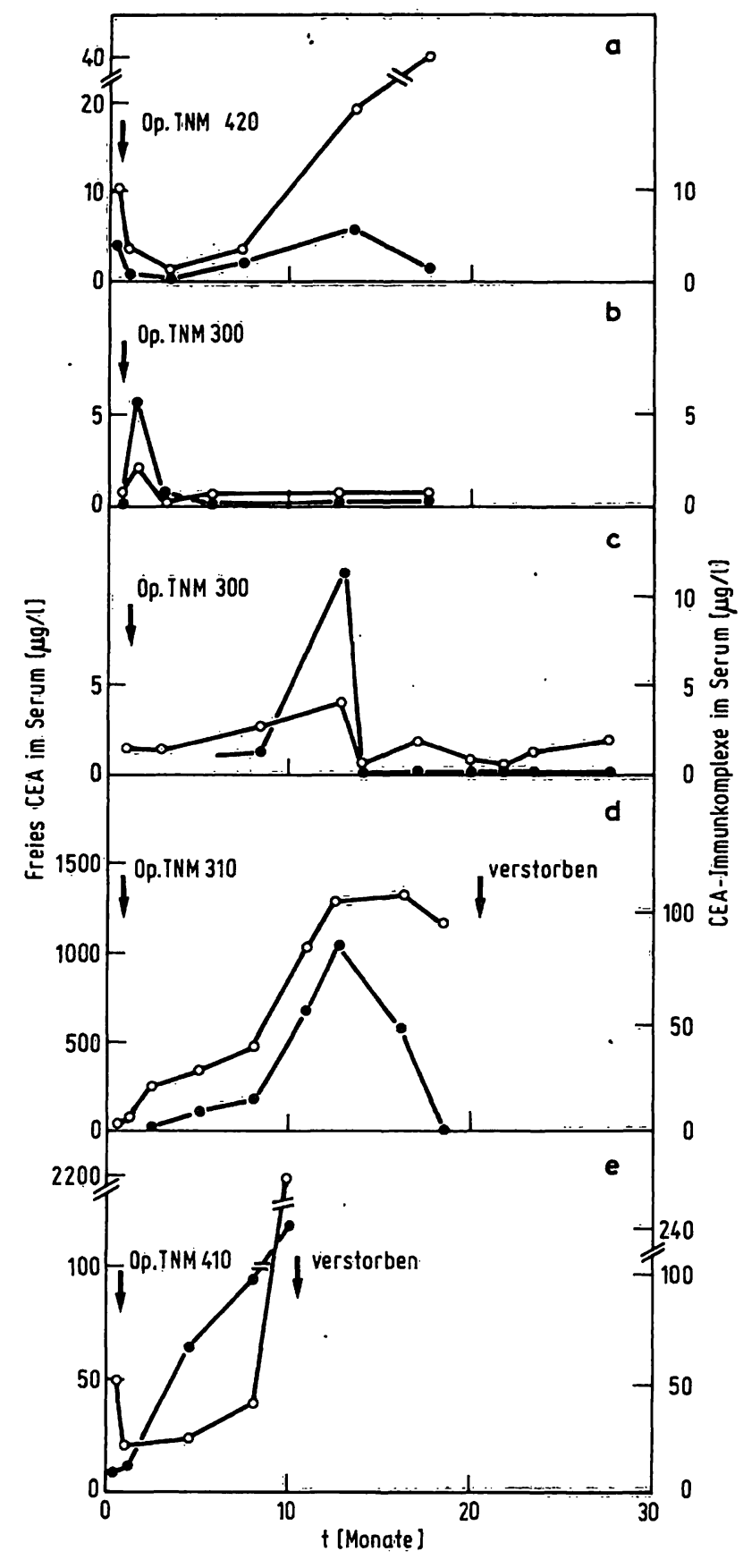

Abb. 1. Beispiel für postoperative Verlaufskurven von freiem CEA ( $(0)$ und CEA-Immunkomplexen $(\bullet)$ bei Patienten mit histologisch gesicherten Adenokarzinomen des Gastrointestinaltrakts:

a) Analoger Verlauf von freiem CEA und CEA-Immunkomplexen;

b) Operations-induzierter Anstieg der CEA-Immunkomplexe und des freien CEA;

c)Intermediärer Anstieg der CEA-Immunkomplex̃e ohne klinischen Anhält für èin Rezidiv;

d)Abfall der CEA-Immunkomplexe vor dem Exitus;

e) Exitus während der Anstiegphase der CEA-Immunkomplexe.

Von besonderem Interesse war die Veränderung der Konzentration der CEA-Immunkomplexe in weï fortgeschrittenen Stadien des Tumorwachstums. Im Verlauf der gegenwärtigen Studie konnten 80 Patienten bis zum Exitus durch Kontrolle der CEA-Immunkómplex: 
Konzentration überwacht werden. Darunter sind auch Patienten, von denen keine präoperative CEA-Immunkomplex-Bestimmung vorlag, d.h. Patienten, die nicht in Tabelle 2 erfaßt sind. In dieser Gruppe waren bei $44 \%$ der Patienten in den Monaten vor dem Exitus CEAImmunkomplexe feststellbar. Bei 13\% der Patienten nahm zunächst die Konzentration der CEA-Immunkomplexe stark zu, um dann wenige Monate vor Exitus auf Null abzusinken, ohne daß sich die Konzentration an freiem CEA wesentlich verminderte. Ein Beispiel für diesen Kurvenverlauf ist in Abbildung 1d wiedergegeben. In $31 \%$ der Fälle jedoch, trat der Exitus schon in der Anstiegsphase der CEA-Immunkomplexe ein (Abb. 1e).

\section{CEA-Immunkomplexe bei Patienten mit Rezidiven}

Fortschreitendes Tumorwachstum kann u.a. dann stattfinden, wenn die zellvermittelte Anti-Tumor-Immunität durch ,blockierende Antikörper" nicht zur vollen Wirkung gelangt (17-21). Das Auftreten von CEAImmunkomplexen bei Rezidiv-Patienten könnte somit einen weiteren Hinweis darauf geben, daß CEA als Tumor-assoziertes Antigen bei der Anti-Tumor-Immunität eine Rolle spielt. In unserer Patientengruppe, bei der postoperativ die Konzentration des freien CEA und der CEA-Immunkomplexe im Serum kontrolliert wurde, sind bisher in 50 Fällen Rezidive festgestellt worden, die von einem Anstieg des freien CEA begleitet waren. Alle diese Patienten waren nach Beurteilung des operativen Situs und den Ergebnissen der histologischen Untersuchung als ,kurativ operiert' eingestuft worden. Bei 30/50 Patienten konnten entweder schon in der ersten Anstiegsphase des freien CEA (16) oder später zum Zeitpunkt der positiven klinischen Diagnose CEAImmunkomplexe nachgewiesen werden, während bei 20/50 Patienten die Rezidivierung nur durch steigende Konzentration an freiem CEA angezeigt wurde.

Die Korrelierung der Rezidivdiagnosen dieser 50 Fälle mit dem Auftreten von CEA-Immunkomplexen ergab einen eindeutigen Trend: Keine CEA-Immunkomplexe konnten in allen 12 Fällen mit lokal begrenżten Rezidiven, in 5/15 Fällen mit peritonealer Karzinose und in 3/23 Fällen mit Lebermetastasen nachgewiesen werden. Daraus ergibt sich, daß mit zunehmender Tumorausdehnung auch der Anțeil der Patienten mit zirkulierenden CEA-Immunkomplexen zunimmt. Die Bestimmung von CEA-Immunkomplexen kann daher auch bei Rezidivierungen für prognostische Aussagen herangezogen werden.

Rezidivierungsprognosen auf der Basis von CEA-Immunkomplexen zum Zeitpunkt der Primärresektion waren bisher nicht möglich. Nach Täbelle 2 ist bei Patienten mit CEA-Immunkomplexen die Tumorausdehnung meist soweit fortgeschritten, daß eine radikale Resektion nicht mehr möglich war. Bei der oben angeführten Gruppe von 50 Rezidiv-Patienten, alle als ,kurativ operiert' eingestuft, waren bei 23 Patienten Bestimmungen der CEA-Immun-

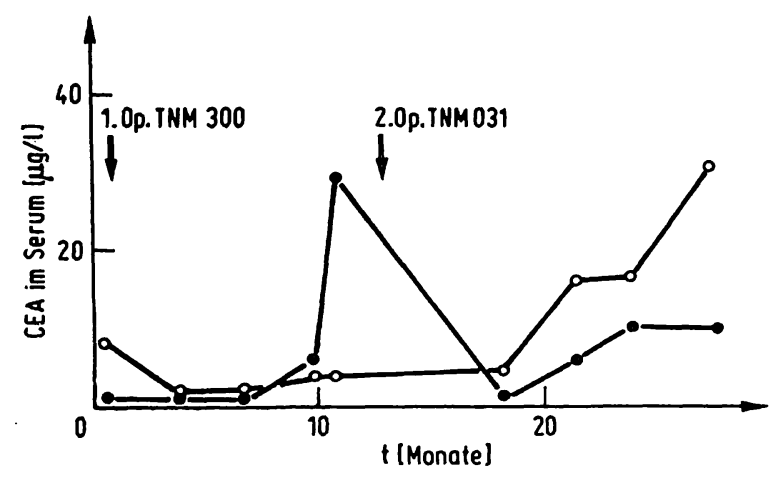

Abb. 2. Verlaufskurve von freiem CEA (o) und CEA-Immunkomplexen ( $\bullet$ ) bei einem Rezidivpatienten. Der Patient hat te ein Adenokarzinom des Colon sigmoideum und entwickelte 10 Monate nach der Primärresektion eine Peritonealkarzinose. In der Second-Look-Operation wurde eine Totalresektion des Omentum majus durchgefuhrt. (Gleicher Maßstab für freies CEA und an Immunglobuline gebundenes CEA).

komplexe auch schon vor der Primärresektion durchgeführt worden. In 20/23 Fällen ließen sich zu diesem Zeitpunkt keine CEA-Immunkomplexe nachweisen. Ein Beispiel einer CEA-Verlaufskurve von freiem CEA und CEAImmunkomplexen bei einem Rezidiv-Patienten ist in Abbildung 2 wiedergegeben. Charakteristisch ist der steile Anstieg der CEA-Immunkomplexe vor der SecondLook-Operation und ihr zunächst vollständiges Verschwinden nach Resektion der Metastasen. Dieses Phänomen ist verschiedentlich für Serumfaktoren beschrieben worden, die eine Anti-Tumorimmunität blockieren können $(31,32)$. Im weiteren Verlauf (Abb. 2) steigen freies CEA und CEA-Immunkomplexe wieder an, so daß bei diesem Patienten fortschreitendes Tumorwachstum angenommen werden muß.

\section{Diskussion}

Die bisherigen Befunde über die Autoantigenität des CEA sind widersprüchlich. Gold et al. (22) und MacSween (23) konnten mit empfindlichen Radioimmuntechniken CEAspezifische Antikörper nachweisen. Anderen Gruppen (33-35) ist es jedoch mit anderen Techniken nicht gelungen, diesen Befund zu bestätigen. Nun muß aber angenommen werden, daß bei einem Überschuß von freiem Antigen im Serum, wie es bei allen Fällen mit pathologisch erhöhten Konzentrationen an CEA der Fall ist, spezifische Antikörper bevorzugt als Immunkomplexe vorliegen. Die meisten der bisher verwendeten Techniken zum Nachweis freier Antikörper sind jedoch ungeeignet, Immunkomplexe nachzuweisen. Die methodischen Kriterien der von Gold et al. (22) und MacSween (23) verwendeten Techniken deuten jedoch darauf hin, daß ihre Befunde möglicherweise nicht auf die Anwesenheit freier anti-CEA-Antikörper, sondern auf CEAImmunkomplexe zurückzuführen sind, da für den positiven Nachweis eine 48 stündige Vorinkubation mit radiomarkiertem CEA nötig war. In unseren voraus- 
gegangenen Untersuchungen (24) konnte die Anwesenheit von freien anti-CEA Antikörpern in Seren von Patienten mit Gastrointestinaltumoren weitgehend ausgeschlossen werden.

Durch andere Autoren wurde über das Auftreten von CEA-Immunkomplexen in glomerulären Ablagerungen bei einem Colonkarzinompatienten mit nephrotischem Syndrom berichtet (36), und schließlich konnten kürzlich CEA-Komplexe mit Immunglobulin $\mathrm{M}$ auch bei einem Patienten mit einem Pankreaskarzinom in der Ascitesflüssigkeit gefunden werden (37). Ferner ließen sich für Patienten mit metastasierenden Colonneoplasmen zu $90 \%$ zirkulierende Immunkomplexe (ohne Angabe der Antigenspezifität) durch C1q-Bindungsteste nachweisen (38).

Die in unserer Studie angesammelten Daten belegen eine weitgehende Korrelation der fortgeschrittenen Stadien der Tumorausdehnung mit dem Auftreten von zirkulierenden CEA-Immunkomplexen. In unserer Studie wurden zum Zeitpunkt der Primärresektion bei $25 \%$ der Patienten (86/350) CEA-Immunkomplexe festgestellt. Dabei waren nur 14/86 Patienten ohne Metastasierung. Bei den Rezidivpatienten $(n=50)$ wurden zum Zeitpunkt der Rezidivdiagnose bei 56\% der Patienten CEA-Immunkomplexe nachgewiesen. Bei allen Patienten mit lokalbegrenzten Rezidiven $(n=12)$ konnten keine CEA-Immunkomplexe entdeckt werden. Das Auftreten von zirkulierenden CEA-Immunkomplexen geht also fast immer mit einer schlechteren Prognose einher.

Die Verlaufskurven der CEA-Immunkomplexe in der Präfinalphase $(1 \mathrm{~d}, e)$ könnten charakteristisch für eine starke Beeinträchtigung der Anti-Tumor-Immunität sein. Wir finden in dieser Patientengruppe immer zunächst einen starken Anstieg der CEA-Immunkomplexe. Hierbei kann häufig die Antikörperproduktion so stark wer den, daß das freie CEA überwiegend als Immunkomplex gebunden wird. Der Exitus kann bereits in dieser Phase eintreten. Bei einigen Patienten jedoch bricht die Antikörperproduktion, möglicherweise in einer Phase der immunologischen Anergie, zusammen, und der Exịtus tritt innerhalb weniger Monate ein.

Der Mechanismus, über den die żirkulierenden CEAImmunkomplexe die Tumorentwicklung beeinflussen können, läßt sich am ehesten durch eine Blockierung der Immunlymphocyten (17) erklären, die bei anderen Systemen bereits beschrieben wurde (18-21) und eine beobachtbare Steigerung des Tumorwachstums auslöste. Dieser Mechanismus muß jedoch nicht $z$ wangsläufig als einzige Ursache für fortschreitendes Tumorwachstum angesehen werden.

Das Problem der Charakterisierung des Immunstatus von Krebspatienten ist durch unsere Befunde nur zu einem kleinen Teil gelöst. Viel wichtiger scheint die Entwicklung von Routine-Methoden, mit denen eine zell-vermittelte Anti-Tumor-Immunität sicher nachgewiesen werden kann und letztlich auch untersucht werden kann, durch welche Faktoren sie beeinflußt wird, um therapeutische Maßnahmen zu optimieren. Entsprechende Untersuchungen sind in Vorbereitung, um insbesondere die Wirkung der CEA-Immunkomplexe bei zell-vermittelter Immunität abzuklären.

\section{Danksagung}

Wir danken Frl. S. Glock für die Mithilfe bei den Radioimmuntesten und Frau $E$. Wehrle für die Durchführung der Datenverarbeitung.

\section{Literatur}

1. Gold, P. \& Freedman, S. O. (1965). J. Exp. Med. 121 , 439-462.

2. Booth, S. N., Jamisson, G. C., King, J. P., Leonard, J., Oates, G. D. \& Dykes, P. W. (1974). Br. Med. J. 4, 183-187.

3. Herrera, M. A., Chu, T. M. \& Holyoke, E. D. (1976). Ann. Surg. 183, 5-9.

4. Holyoke, E. D., Chu, T. M. \& Murphy, G. P. (1975). Cancer 35, 830-836.

5. Lo Gerfo, P. \& Herter, F. P. (1975). Ann. Surg. 181, 81-84.

6. Mach, J. P., Jaeger, P. H., Bertholt, M. M., Ruegsegger, C. H., Loosli, R. M. \& PeHavel, J. (1974). Lancet II, 535 -540.

7. MacKay, A. M., Patel, S., Carter, S., Stevens, U., Laurence, D. J. R., Cooper, E. H. \& Neville, A. M. (1974). Br. Med. J. 4, $382-385$

8. Martin, E. W., James, K. K., Hurtubise, P. E., Catalano, P. \& Minton, J. P. (1977). Cancer 39, 440-446.

9. Ravry, M., Moertel, C. G., Schutt, A. J. \& Go, V. L. W. (1974). Cancer 34, 1230-1234.

10. Sorokim, J. J., Sugarbaker, P. H., Zamcheck, N., Pisick, M. Kupchick, H. Z. \& Moore, F. D. (1974). J. Am. Med. Ass. 288, 49-53.
11. Staab, H. J., Anderer, F. A., Stumpf, E. \& Fischer, R. (1977). Dtsch. Med. Wochenschr. 102, 1082-1086.

12. Sugarbaker, P. H., Skarin, A. T. \& Zamcheck, N. (1976). J. Surg. Oncol. 8, $523-537$.

13. Ratcliffe, J. G., Wood, C. B., Burt, R. W., Malcolm, A. J. H. \& Blumgart, L. H. (1978). Proc. of the 6 th IRGCP-meeting. Carcinoembryonic Proteins, Vol. II, p. 75-80 (Lehmann, F. G. ed.) Elsevier North Holland Biomedical Press, Amsterdam.

14. Staab, H. J., Anderer, F. A., Stumpf, E. \& Fischer, R. (1978). Scand. J. Immunol. 8, Suppl. 8, 459-464.

15. Staab, H. J., Anderer, F. A., Stumpf, E. \& Fischer, R. (1978). J. Surg. Oncol. 10, 273-282.

16. Staab, H. J., Anderer, F. A., Stumpf, E. \& Fischer, R. (1978). Am. J. Surgery 136, 322-327.

17. Hellström, I., Sjögren, H. O., Warner, G. A. \& Hellström, K. E. (1971). Int. J. Cancer 7, 226-237.

18. Baldwin, R. W., Price, M. R. \& Robins, R. A. (1972). Nature New Biol. 238, 185-187.

19. Jose, D. G. \& Seshadri, R. (1974). Int. J. Cancer 13, 824838. 
20. Sjögren, J. O., Hellström, I., Bansal, S. C. \& Hellström, K. E. (1971). Proc. Nat. Acad. Sci. USA 68, 1372-1375.

21. Sjögren, H. O., Hellström, I., Bansal, S. C., Warner, G. A. \& Hellström, K. E. (1972). Int. J. Cancer 9, 274-283.

22. Gold, J. M. Freedman, S. O. \& Gold, P. (1972). Nature New Biol. 239, 60-62.

23. MacSween, J. M. (1975). Int. J. Cancer 15, 246-252.

24. Kapsopoulou-Dominos, K. \& Anderer, F. A. (1979). Clin. Exp. Immunol. 35, 190-195.

25. Kapsopoulou-Dominos, K. \& Anderer, F. A. (1979). Clin. Exp. Immunol., 37, 25-32.

26. Hansen, H. J., Lance, R. P. \& Krupey, J. (1971). Abstract. Clin. Res. 19, 143.

27. Primus, F. J., Newman, E. S. \& Hansen, H. J. (1977). J. Immunol. 118, 55-61.

28. Burtin, P. (1978). Ann. Immunol. (Inst. Pasteur) $129 C$, $185-198$.

29. International Union against Cancer; TNM-Klassifizierung der malignen Tumoren und allgemeine Regeln zur Anwendung des TNM-Systems (1970). Springer, Berlin-HeidelbergNew York.

30. Spiessl, B. (1976). Z. Allgemeinmed. 52, 1133-1138.

31. Hellström, I., Hellström, K. E. \& Sjögren, H. O. (1970). Cell. Immunol. 1, 18-30.

32. Maluish, A. \& Halliday, W. J. (1974). J. Natl. Cancer Inst. $52,1415-1420$.

33. Collatz, E., von Kleist, S. \& Burtin, P. (1971). Int. J. Cancer 8, 298-303.

34. LoGerfo, P., Herter, F. D. \& Bennett, F. J. (1972). Int. J. Cancer 9, 344-348.

35. Sorokim, J. J., Kupchick, H. Z. \& Zamcheck, N. (1973). J. Natl. Cancer Inst. 51, 1081-1083.

36. Costanza, M. E., Pinn, V., Schwartz, R. S. \& Nathanson, L. (1973). New Engl. J. Med. 289, 520-522.

37. Harvey, S. R., van Dusen, L. R., Douglass, H. O., Holyoke, E. D. \& Chu, T. M. (1978). J. Natl. Cancer Inst. 61, 11991203.

38. Rossen, R. D. (1978). Antibiotics Chemother. 22, 5.

Dr. H. J. Staab, Prof. Dr. F. A. Anderer Friedrich-Miescher-Laboratorium der Max-Planck-Gesellschaft Spemannstraße 37-39 D-7400 Tübingen

Dr. E. Stumpf, Prof. Dr. R. Fischer Chirurgische Klinik des Städtischen Krankenhauses Bad Cannstatt Theodor-Veiel-Str. 90 D-7000 Stuttgart 50 
, 